\title{
CEREBROSPINAL FLUID BRAIN DERIVED NEUROTROPHIC FACTOR IN THE CHILDREN
} WITH BACTERIAL MENINGITIS

\author{
F. Mashayekhi ${ }^{1}$, E. Mirzajani ${ }^{2}$
}

${ }^{I}$ Department of Biology, University of Guilan, ${ }^{2}$ Department of Biochemistry, Guilan Medical Sciences University, Rasht, Iran

Meningitis is an inflammation of the membranes that surround the brain and spinal cord, thereby involving the arachnoid, the pia mater and the interposed cerebrospinal fluid (CSF). The examination of CSF samples may provide information about causative microorganism. CSF contains growth factors including brain derived growth factor (BDNF) which is present under specific pathological and physiological conditions. As CSF is in close contact with the extracellular space of the brain, biochemical brain modifications could be reflected in the CSF and analysis of cytokines and growth factors might identify biomarkers of meningitis. Thus, it is important to study the biochemistry of CSF in order to find a reliable biomarker. The aim of this study was to analyze the level of BDNF in the CSF of the children with bacterial meningitis using ELISA. A total of 66 samples of CSF from normal and meningitis were collected by lumbar puncture. BDNF concentration in the CSF of normal subjects and patients with bacterial meningitis was measured using enzyme linked immunosorbent assay (ELISA). It was shown that the concentration Of BDNF in the CSF samples with meningitis was higher than in normal CSF. The mean BDNF concentration in CSF of patients with meningitis amounted to $49.22 \pm 7.12 \mathrm{pg} / \mathrm{ml}$, which was significantly higher than that of normal, i.e. 9.15 $\pm 2.26 \mathrm{pg} / \mathrm{ml}$. In conclusion, the data from this study suggests that BDNF could be involved in the pathophysiology of meningitis. The results of our study indicate that BDNF may have neuroprotective properties in meningitis. 